\title{
Repurposing Clinical Drugs as AdoMetDC Inhibitors Using the SCAR Strategy
}

\author{
Yan Zhang ${ }^{1,2+}$, Qiang Zheng ${ }^{1,2+}$, Yin Zhou ${ }^{1,2}$ and Sen Liu ${ }^{1,2 * \neq}$ \\ 'National "111" Center for Cellular Regulation and Molecular Pharmaceutics, Key Laboratory of Industrial Fermentation \\ (Ministry of Education), Hubei University of Technology, Wuhan, China, ${ }^{2}$ Institute of Biomedical and Pharmaceutical \\ Sciences, Hubei Key Laboratory of Industrial Microbiology, Hubei University of Technology, Wuhan, China
}

\section{OPEN ACCESS}

Edited by:

Manuela Marcoli,

University of Genoa, Italy

Reviewed by:

Simone Brogi,

University of Pisa, Italy

Otto Phanstiel,

University of Central Florida,

United States

${ }^{*}$ Correspondence:

Sen Liu

senliu.ctgu@gmail.com

tThese authors have contributed

equally to this work

FORCID:

Sen Liu

orcid.org/0000-0001-5182-7241

Specialty section:

This article was submitted to

Experimental Pharmacology and Drug Discovery,

a section of the journal

Frontiers in Pharmacology

Received: 31 December 2019

Accepted: 24 February 2020

Published: 11 March 2020

Citation:

Zhang Y, Zheng Q, Zhou Y and Liu S (2020) Repurposing Clinical Drugs as AdoMetDC Inhibitors Using the SCAR Strategy.

Front. Pharmacol. 11:248. doi: 10.3389/fphar.2020.00248
With the escalating costs in drug development, discovering new uses of approved drugs, i.e., drug repurposing, has attracted increasing interest. Spermidine and spermine are important polyamines for most cells and their biosynthesis are strictly regulated by the polyamine metabolic network. In cancerous cells and tumor environments, the concentrations of polyamines are much higher than in normal cells. During the synthesis of spermidine and spermine, an amino-propyl group is provided by decarboxylated S-adenosylmethionine, and the latter is generated from S-adenosylmethionine by AdoMetDC (AdoMet decarboxylase). Therefore, as a ratelimiting enzyme in the biosynthesis of spermidine and spermine, AdoMetDC has been an attractive drug target in cancer studies. In the last decades, many AdoMetDC inhibitors have been discovered, and several AdoMetDC inhibitors are under clinical trials, but unfortunately, none of them have been approved yet. To overcome the high costs in time and money for discovering de novo inhibitors, we set out to repurpose clinic drugs as AdoMetDC inhibitors. We used steric-clashes alleviating receptors (SCAR), a computer-aided drug discovery strategy developed by us recently for in silico screening. By combining computational screening and experimental validation, we successfully identified two approved drugs that have inhibitory potency on AdoMetDC's enzymatic activity. SCAR was previously shown to be suitable for the discovery of both covalent and non-covalent inhibitors, and this work further demonstrated the value of the SCAR strategy in drug repurposing.

Keywords: polyamines, drug repurpose, drug discovery, computer-aided drug design, AdoMet decarboxylase

\section{INTRODUCTION}

Accompanying extended human lifespans and deteriorating environment, the world is facing increasing disease burdens from cancer, mental diseases, virus infections, etc. (Chang et al., 2019). Drugs have been proven to be indispensable for combating diseases and improving life quality, but the number of new drugs brought to the market has been declining (Scannell et al., 2012). One reason for this trend is that the costs of drug discovery have continued to increase. In the traditional drug development process, it costs over 15 years and one billion US dollars from target identification, target validation, hit discovery, and lead optimization to preclinical and clinical trials before a compound gets approved (Kaitin, 2010). Even more deadly to nascent pharmaceutical 
companies is that even if a compound enters phase II clinical trials, it has merely a $10 \%$ chance of getting approved due to unexpected human toxicity and lack of efficacy (Hodos et al., 2016). Therefore, saving costs in time, preclinical development, and even some or all clinical trials in drug development is extremely attractive, which is the main reason that drug repurposing (also known as drug repositioning, reprofiling, redirecting, or rediscovering) has attracted increasing attention in recent years (Baker et al., 2018). Drug repurposing identifies new uses for a drug beyond its original use, therefore, the data for human pharmacokinetics, safety, as well as the preclinical results, are readily available. This advantage makes it easier to get the new uses of the drug approved. Representative examples of drug repurposing include the sildenafil for erectile dysfunction and the anti-cancer use of thalidomide (Liu et al., 2013). Therefore, drug repurposing represents a most promising field in drug development (Cha et al., 2018).

Polyamines are small cationic compounds that exist in nearly all cells (Miller-Fleming et al., 2015). In mammalian cells, the most common polyamines are putrescine, spermidine, and spermine. The structures of these polyamines are rather simple, with linear aliphatic chains bridged or flanked by amino groups, however, they can affect the structures and functions of nucleic acids, proteins, and membranes (Miller-Fleming et al., 2015). Therefore, polyamines are indispensable players in regulating cellular signaling modules and cell fates. The cellular concentrations of these polyamines could be up to $20 \mathrm{mM}$ ( $\mathrm{Li}$ et al., 2019), which is strictly tuned by a complicated metabolic network including biosynthesis, interconversion, metabolism, and membrane transportation (Pegg, 2009). It has been well demonstrated that the proliferation of cancer cells requires higher cellular polyamine levels than normal cells, so the polyamine metabolic network in cancer cells is dysregulated to increasing the biosynthesis or membrane uptake of these polyamines (Casero, 2011). Consequently, targeting the polyamine metabolic network to inhibit the biosynthesis and/or membrane uptake of polyamines is a promising anti-cancer strategy (Casero, 2011).

In mammalian cells, putrescine is the precursor of spermidine and spermidine is the precursor of spermine. During these conversions, an amino-propyl group from decarboxylated S-adenosylmethionine (dcAdoMet) is transferred (Figure 1A). The production of dcAdoMet is generated from the decarboxylation of S-adenosylmethionine (AdoMet), which is catalyzed by AdoMetDC (AdoMet decarboxylase) (Figure 1A). Therefore, AdoMetDC is a rate-limiting enzyme in the biosynthesis of polyamines (Liao et al., 2015). To inhibit polyamine synthesis, many AdoMetDC inhibitors have been developed, including the first-generation inhibitor methylglyoxal bis (guanylhydrazone) (MGBG), the second-generation inhibitor SAM486A (Sardomozide, also known as CGP48664), and the third-generation inhibitor AbeAdo (5'-([(Z)-4-amino-2butenyl]methylamino)-5'-deoxy-adenosine), etc. (Casero, 2011). Several AdoMetDC inhibitors have been tested in clinical trials treating cancers (Herr et al., 1986; Paridaens et al., 2000), but none of them has been finally approved for clinical use due to low efficiency or strong side-effects. Therefore, discovering new AdoMetDC inhibitors will be highly valuable.
Recently, we described the computer-aided discovery of both non-covalent and covalent AdoMetDC inhibitors based on the in silico protein design strategy (Liao et al., 2015; Ai et al., 2016). To assist the discovery of covalent AdoMetDC inhibitors, we presented a docking strategy named as steric-clashes alleviating receptors (SCAR) (Ai et al., 2016). Additionally, we demonstrated that this strategy also works good for evaluating non-covalent inhibitors (Ai et al., 2016). In this work, we set out to test the use of the SCAR method in repurposing the drugs in clinical trials as AdoMetDC inhibitors. We successfully identified two drugs worth further investigation. Our results showed that the SCAR method is an attracting strategy for both de novo drug discovery and drug repurposing.

\section{MATERIALS AND METHODS}

\section{Structure Preparation of the Small Molecules}

The $3 \mathrm{D}$ structures of the small molecules were downloaded as mol2 files from $\mathrm{ZINC1}^{1}$ (Sterling and Irwin, 2015). In ZINC15, the $3 \mathrm{D}$ conformations were protonated at physiological $\mathrm{pH}$ and biologically relevant tautomers were generated for each molecule, which resulted in more than one conformation for many molecules. From these mol2 files, MGLTools (version 1.5.6) was used to generate the PDBQT files for docking.

\section{Structure Preparation of the Protein}

The AdoMetDC structure (PDB ID: 3DZ5) was downloaded from $\mathrm{PDB}^{2}$. The Pyr68 residue of the alpha-chain of AdoMetDC was eliminated as previously mentioned (Ai et al., 2016) before the structure was energetically minimized using the relax protocol in Rosetta (Leaver-Fay et al., 2011). The options for minimization were: -relax:constrain_relax_to_start_coords -relax:coord_constrain_sidechains -relax:ramp_constraints false -s protein.pdb -ex1 -ex2 -use_input_sc -flip_HNQ -no_optH false. Finally, MGLTools (version 1.5.6) was used to generate the PDBQT file for docking.

\section{In silico Docking}

The computational docking of the small molecules to AdoMetDC was similar with the process previously described (Ai et al., 2016). Briefly, the substrate binding pocket of AdoMetDC was used as the reference to define a grid box in MGLTools. Then, AutoDock Vina (version 1.1.2) was used to dock small molecules to the indicated grid box of AdoMetDC. The exhaustiveness parameter was set as 100 to extensively search possible docking conformations. For each ligand, up to 20 conformations were output. Finally, the docked conformations were then ranking by docking scores. After docking, we used the following rules to filter the docking results: (1) The RMSD cutoff was set as 3.0 compared to the first conformation, (2) the score cutoff was set as -8.0 (Ai et al., 2016), and (3) for each compound, $75 \%$ or more output conformations fulfilled these two cutoffs.

\footnotetext{
${ }^{1}$ http://zinc15.docking.org

${ }^{2}$ http://www.rcsb.org
} 
A

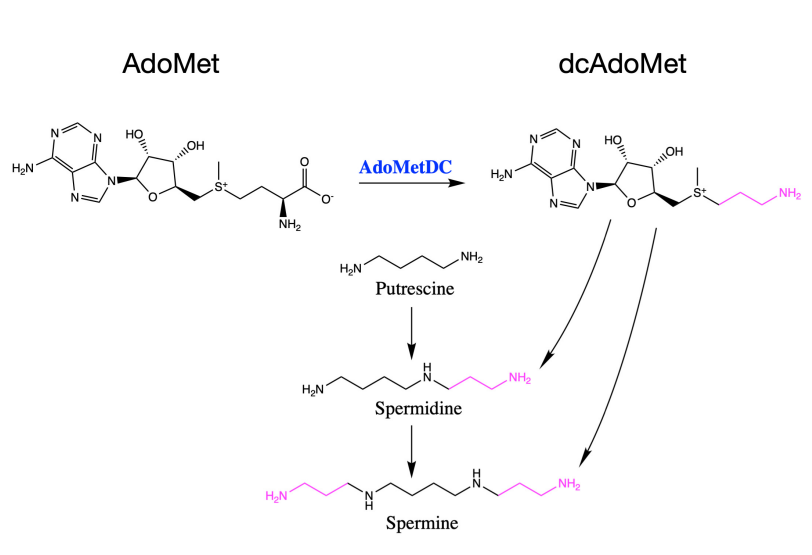

B

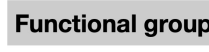

$-\mathrm{O} \backslash \mathrm{NH}_{2}$<smiles>COC(=O)C(C)N</smiles><smiles>CNC(=N)N</smiles><smiles>CNN</smiles><smiles>C=CCN</smiles><smiles>CC(=O)NN</smiles>

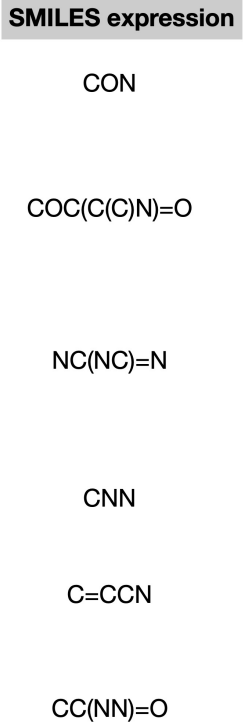

$\mathrm{CC}(\mathrm{NN})=\mathrm{O}$

FIGURE 1 | AdoMetDC is a rate-limiting enzyme for polyamine synthesis and several functional groups repeatedly occur in AdoMetDC inhibitors. (A) The reaction catalyzed by AdoMetDC is from AdoMet to decarboxylated AdoMet (dcAdoMet), which provides the amino-propyl group for the biosynthesis of spermidine and spermine. (B) The functional groups observed in previous AdoMetDC inhibitors were used for in silico screening in this study. The SMILES expressions used for library filtering are shown next to the structure.

Additionally, to minimize the numbers of the out-of-pocket atoms, an affinity density was defined as the average score of the non-hydrogen atoms in the compound and set as -0.28 (lower is better). After that, the docked conformations were manually checked to evaluate the structural similarity and the position of the functional groups.

\section{Compounds and Materials}

The compounds used for experimental screening were purchased from the following commercial vendors: ZINC-000001530713 and ZINC-000006482036 from MedChemExpress'; ZINC000043195697 from Selleck Chemicals ${ }^{4}$; ZINC-000043205655, ZINC-000100055899, and ZINC-000144542146 from Topscience ${ }^{5}$. The compounds were fully dissolved in DMSO to prepare $60 \mathrm{mM}$ stock solutions. The carbon dioxide kit was purchased from BioSino Bio-Technology and Science Inc. (Beijing, China).

\section{Protein Expression and Purification}

The expression and purification of human AdoMetDC were similar as mentioned before (Liao et al., 2015; Ai et al., 2016). Briefly, the coding sequence of AdoMetDC was inserted in pET15b, and the plasmid was transformed into the Escherichia coli strain BL21(DE3). The expression of AdoMetDC was induced by $0.5 \mathrm{mM}$ of IPTG (isopropyl $\beta$-d1-thiogalactopyranoside) at $15^{\circ} \mathrm{C}, 250 \mathrm{rpm}$ for $12 \mathrm{~h}$. The cells

${ }^{3}$ https://www.medchemexpress.com

${ }^{4}$ http://www.selleckchem.com

${ }^{5}$ http://www.topscience.cn were collected, resuspended, and broken by high-pressure homogenizer in the cell lysis buffer $\left(20 \mathrm{mM} \mathrm{Na}_{2} \mathrm{HPO}_{4}, 500 \mathrm{mM}\right.$ $\mathrm{NaCl}, \mathrm{pH} 7.0,2.5 \mathrm{mM}$ putrescine, $30 \mathrm{mM}$ imidazole). The solution was centrifuged, and the supernatant was loaded in a Ni-NTA gravity column for the purification of His-tagged AdoMetDC. Finally, the protein was purified with a Sephacryl S-200 HR column (GE Healthcare) and eluted with the elution buffer $(300 \mathrm{mM} \mathrm{NaCl}, 0.1 \mathrm{mM}$ EDTA, $2.5 \mathrm{mM}$ DTT, $1 \mathrm{mM}$ HEPES, pH 7.0). The purified AdoMetDC was used for the activity analyses without removing the His tag.

\section{AdoMetDC-PEPC-MDH Assay}

The AdoMetDC-PEPC-MDH assay was developed in our previous study (Liao et al., 2015) and used to evaluate the activity of AdoMetDC with the procedures similar as before (Liao et al., 2015; Ai et al., 2016). Briefly, AdoMetDC was mixed with DMSO, MGBG or compounds, respectively, in wells and incubated at $37^{\circ} \mathrm{C}$ for $30 \mathrm{~min}$ before R2 [400 unit/L phosphoenolpyruvate carboxylase (PEPC), 600 unit/L malate dehydrogenase (MDH), $0.45 \mathrm{mM} \mathrm{NADH}$ ] was added to the wells in a 96-well plate. In another row, R1 [7.0 mM phosphoenolpyruvate (PEP), $8.0 \mathrm{mM} \mathrm{MgCl}_{2}$ ] and S-adenosylmethionine (AdoMet) were mixed. The plate was incubated in a $37^{\circ} \mathrm{C}$ incubator for $5 \mathrm{~min}$, and the reaction was initiated by mixing the two solutions in these two wells. The absorbance data at $340 \mathrm{~nm}$ was recorded every $30 \mathrm{~s}$ at $37^{\circ} \mathrm{C}$ on a multifunctional microplate reader (BioTek Synergy $\mathrm{H} 1)$ for $10 \mathrm{~min}$. The final reaction mixture contained $120 \mu \mathrm{L} \mathrm{R} 1$, $40 \mu \mathrm{L}$ R2, 1 mM AdoMet, $1 \mu \mathrm{M}$ AdoMetDC, and $100 \mu \mathrm{M}$ MGBG or compounds. For each compound, at least three independent experiments were performed. 


\section{HPLC (High Performance Liquid Chromatography) Assay}

The compounds were incubated with AdoMetDC in the reaction buffer $\left(20 \mathrm{mM} \mathrm{Na}_{2} \mathrm{HPO}_{4}\right.$, pH $\left.7.0,100 \mathrm{mM} \mathrm{NaCl}\right)$ at $37^{\circ} \mathrm{C}$ for $30 \mathrm{~min}$. AdoMet was added to initiate the reaction. The final reaction volume was $200 \mu \mathrm{L}$, containing $100 \mu \mathrm{M}$ inhibitors, $1 \mu \mathrm{M}$ AdoMetDC, and $1 \mathrm{mM}$ AdoMet. At the indicated time points, the reaction was stopped by $800 \mu \mathrm{L}$ of methanol. The samples were centrifuged at $20,000 \mathrm{~g}$ for $5 \mathrm{~min}$, and the supernatants were analyzed. The analysis was performed using a reverse-phase column (Waters C18 column, $5 \mu \mathrm{m}, 4.6 \times 250 \mathrm{~mm}$ ) on a Dionex Ultimate 3000 system (Thermo Scientific) at $30^{\circ} \mathrm{C}, 254 \mathrm{~nm}$. The mobile phase was $10 \mathrm{mM}$ ammonium format ( $\mathrm{pH} \mathrm{3.5}$, adjusted with formic acid) and methanol $(97: 3, \mathrm{v} / \mathrm{v})$, and the flow rate was $0.7 \mathrm{~mL} / \mathrm{min}$. The data were collected and the peak areas were integrated in the Chromeleon software. For each compound, at least three independent experiments were performed.

\section{Mass Spectrometry Analysis}

The compounds were used for experimental screening. Samples were prepared and analyzed following the previous method (Ai et al., 2016). Briefly, the inhibitors were incubated with AdoMetDC in the reaction buffer $\left(20 \mathrm{mM} \mathrm{Na}_{2} \mathrm{HPO}_{4}, \mathrm{pH} 7.0\right.$, $100 \mathrm{mM} \mathrm{NaCl}$ ) at $37^{\circ} \mathrm{C}$ for $120 \mathrm{~min}$. The final reaction volume was $200 \mu \mathrm{L}$, including $100 \mu \mathrm{M}$ inhibitors and $10 \mu \mathrm{M}$ AdoMetDC. The matrix-assisted laser desorption ionization time-of-flight mass spectrometry (MALDI-TOF-MS) analysis was performed on an Applied Biosystems 5800 (AB SCIEX, Concord, Canada) equipped with a $355 \mathrm{~nm} \mathrm{Nd:YAG} \mathrm{laser} \mathrm{source.} \mathrm{SA} \mathrm{matrix} \mathrm{(sinapic}$ acid) was prepared in $30 \%$ acetonitrile (ACN) aqueous solution. The acquired data were processed in AB Sciex Data Explorer v4.5 (AB SCIEX, Concord, Canada). For each compound, at least three independent experiments were performed.

\section{RESULTS}

\section{Database Preparation for In silico Docking}

We were interested in investigating if any in-trial drugs are potential AdoMetDC inhibitors. Therefore, we obtained the in-trials compound library from the ZINC database ${ }^{6}$, which contains 5,811 compounds. Among these compounds, 3,447 are approved drugs by the Food and Drug Administration (FDA) of United States or other major juridications, and another 2,364 compounds are drugs in clinical trials (Sterling and Irwin, 2015). A subset docking library was prepared from this compound library by filtering the compounds with the functional groups of AdoMetDC inhibitors defined in our previous work (Ai et al., 2016). Both non-covalent and covalent functional groups were used in this step (Figure 1B), since we wanted to search for both covalent and non-covalent AdoMetDC inhibitors. The pre-defined functional groups were represented by their simplified molecular input line entry system (SMILES)

${ }^{6}$ http://zinc15.docking.org expressions as below: $\mathrm{CON}, \mathrm{COC}(\mathrm{C}(\mathrm{C}) \mathrm{N})=\mathrm{O}, \mathrm{NC}(\mathrm{NC})=\mathrm{N}$, $\mathrm{CNN}, \mathrm{C}=\mathrm{CCN}, \mathrm{CC}(\mathrm{NN})=\mathrm{O}$. Finally, 613 compounds were included in the docking library. Furthermore, the different conformations of these compounds were downloaded from ZINC15 as is (Sterling and Irwin, 2015), resulting in a docking library containing 1,074 entities.

\section{SCAR Screening of Potential AdoMetDC Inhibitors}

Previously, our group set up a SCAR strategy for screening covalent AdoMetDC inhibitors (Ai et al., 2016). As demonstrated in the previous work (Ai et al., 2016), this method was able to recapture the $\mathrm{X}$-ray conformations of both covalent and non-covalent AdoMetDC inhibitors. Therefore, we adopted this method for in silico screening in this work. Similar as described in our previous work (Ai et al., 2016), a high-resolution AdoMetDC structure (PDB ID: 3DZ5) was used as the target. To perform SCAR docking, the Pyr68 residue of the alphachain of AdoMetDC was wholly eliminated (Figure 2A), and the structure was energetically minimized in Rosetta (LeaverFay et al., 2011). The substrate binding pocket in AdoMetDC was defined as the search region, and the compound entities in the prepared docking library were docked to this pocket one by one. Following the docking process, the candidate ligands were evaluated by the rules as described in Materials and Methods. At last, six compounds were chosen and available for purchase to do experimental validation (Figure 2B). Meanwhile, SAM486A was also identified as a candidate during this computational process, but it was not included in further experimental tests since it is a known AdoMetDC inhibitor.

\section{Experimental Validation of Potential AdoMetDC Inhibitors}

We used the AdoMetDC-PEPC-MDH assay developed in our previous study (Liao et al., 2015) to experimentally validate the inhibitory potency of the purchased compounds on AdoMetDC's enzymatic activity. This assay quantifies the generation of $\mathrm{CO}_{2}$ from the decarboxylation reaction and is suitable for the initial semi-quantitative screening. As shown in Figure 3A, among the six compounds, ZINC000043195697 and ZINC000144542146 slightly inhibited AdoMetDC's activity in the first-round fast screening. As of ZINC6482036 and ZINC43205655, we noticed that their light absorbance values were distorted by their low solubility and their inhibitory effects were not reliable (data not shown). This was not unexpected since the AdoMetDCPEPC-MDH assay is fast but prone to interferences. Next, a more careful evaluation was repeated to confirm the inhibitory potency of ZINC000043195697 and ZINC000144542146. The data (Figures 3B,C) showed that ZINC000043195697 was better than ZINC000144542146, although they were both less potent than MGBG.

Following the quick screening step using the AdoMetDCPEPC-MDH assay, we continued to verify the inhibitory potency of ZINC000043195697 and ZINC000144542146 more precisely. To this end, we performed an HPLC assay, which quantifies the consumption of the substrate AdoMet 


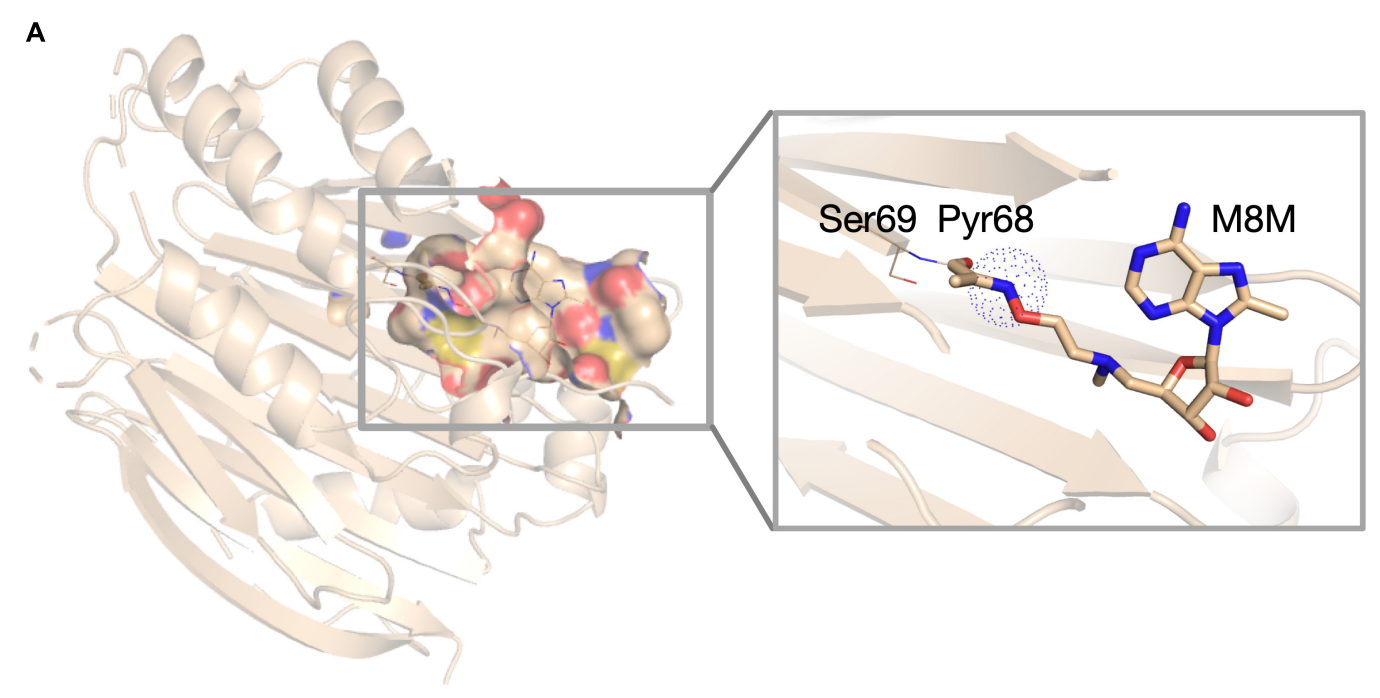

B<smiles>CC(C)[C@H](N)C(=O)OCCOCn1cnc2c(=O)[nH]c(N)nc21</smiles>

ZINC000001530713<smiles>CCOC(=O)C(N)Cc1ccc(-c2cc(OC(c3ccc(Cl)cc3-n3ccc(C)n3)C(F)(F)F)nc(N)n2)cc1</smiles>

ZINC000043205655<smiles>CO/N=C(/N)c1ccc(C2CCC(c3ccc(/C(N)=N\OC)cc3)O2)cc1</smiles>

ZINC000006482036<smiles>CC(C)OC(=O)[C@H](C)NP(=O)(CO[C@H](C)Cn1cnc2c(N)ncnc21)Oc1ccccc1</smiles>

ZINC000100055899<smiles>COc1cc2c(cc1OC)[C@H]1C[C@H](OC(=O)C(N)C(C)C)[C@H](CC(C)C)CC1CC2</smiles>

ZINC000043195697<smiles>CN1C(=N)N[C@](C)(c2cc(NC(=O)c3ccc(F)cn3)ccc2F)CS1(=O)=O</smiles>

ZINC000144542146

FIGURE 2 | The in silico screening of potential AdoMetDC inhibitors. (A) The covalent inhibitor M8M is covalently attached with Pyr68 in AdoMetDC (PDB ID: 3DZ5). For SCAR docking, the Pyr68 of AdoMetDC was computationally removed, and the dotted sphere was defined as the covalent center to evaluate if a ligand is a potential covalent inhibitor as previously described (Ai et al., 2016). (B) Six compounds were chosen from the in silico screen for experimental validation. The ZINC IDs were shown below the corresponding structures. All structural figures in this paper were prepared in Pymol.

during the reaction. The HPLC result (Figure 3C) confirmed that ZINC000043195697 obviously inhibited AdoMetDC's activity and ZINC000144542146 could not significantly inhibit AdoMetDC.

\section{Analysis of the Binding Structure and Mechanism}

To analyze the binding mechanism of the identified inhibitors with AdoMetDC, we looked into the docked conformations. For ZINC000043195697, the first docking conformation is shown in Figure $\mathbf{4 A}$ and the best docking conformation with a possible functional amino group close to the covalent center is shown in Figure 4B. Both poses form preferred $\pi-\pi$ and/or cation $-\pi$ interactions with Phe7 and Phe223 of AdoMetDC (Ai et al., 2016). However, the first pose (Figure 4A) has a hydroxyl group that will conflict with Pyr68 (not shown) since this group cannot form covalent bonds with Pyr68. For the second pose (Figure 4B), the amino group is not placed very well in the covalent sphere, so this might affect the formation of covalent bonds and the binding affinity. For ZINC000144542146, the first docking conformation is shown in Figure 4C, which was also a conformation with a potential functional amino group in the covalent sphere. The backbone phenyl ring also forms $\pi-\pi$ interactions with Phe7 and Phe223 of AdoMetDC. Therefore, we continued to test if these two compounds could covalently bind AdoMetDC. As previously described (Ai et al., 2016), a MALDI-TOF-MS analysis was performed. However, the data (Figure 4D) showed that no ligands were attached to the protein and caused significant increases in the mass of either the alpha chain (theoretical Mw: $30655 \mathrm{Da}$ ) or the whole protein (theoretical Mw: $40635 \mathrm{Da}$ ), indicating that these compounds should not be AdoMetDC's covalent inhibitors. 


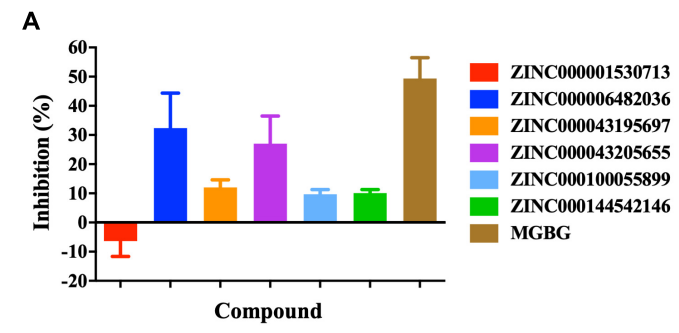

C

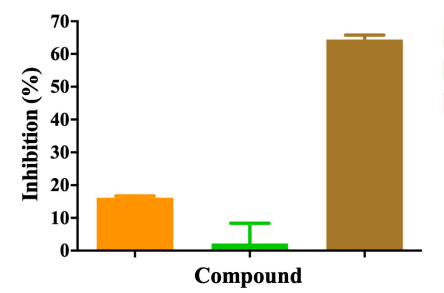

B

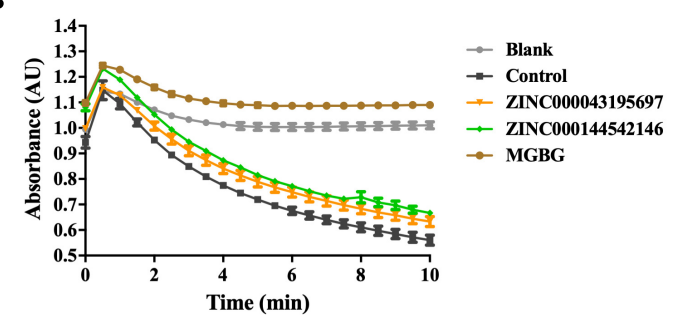

D

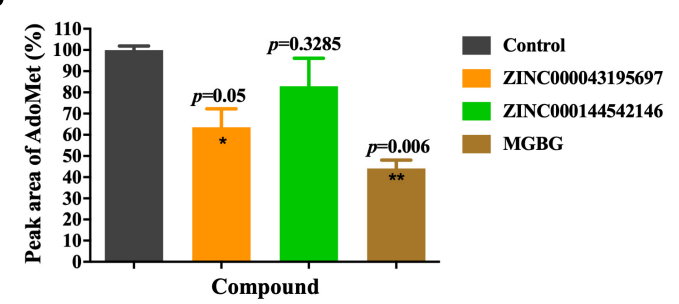

FIGURE 3 | The experimental validation of the inhibitory efficacy of the purchased compounds. (A) The inhibitors were evaluated by the AdoMetDC-PEPC-MDH assay (Liao et al., 2015) quantifying the released $\mathrm{CO}_{2}$ from decarboxylation. The absorbance changes $(\triangle \mathrm{AU}$ ) in the first 5 min were used to calculate the inhibition percentages compared to the control without inhibitors. A note is although ZINC6482036 and ZINC43205655 showed high inhibition percentages in this assay, they were found to be false positives in the HPLC assay. (B) ZINC000043195697 and ZINC000144542146 were tested in the second-phase evaluation with the AdoMetDC-PEPC-MDH assay. (C) The calculated inhibition rates from the data in (B). (D) The inhibitors were evaluated by the HPLC assay quantifying the consumption of AdoMet during the reaction. The amounts of AdoMet were quantified by calculating the peak areas and normalized against the control. The control was the sample without AdoMetDC added. All compounds were tested at $100 \mu \mathrm{M}$. The data are shown as mean \pm SEM $(n=3)$, and the Student's $t$-test was performed for statistical analysis. ${ }^{*} p \leq 0.05 ;{ }^{* *} p \leq 0.01$.

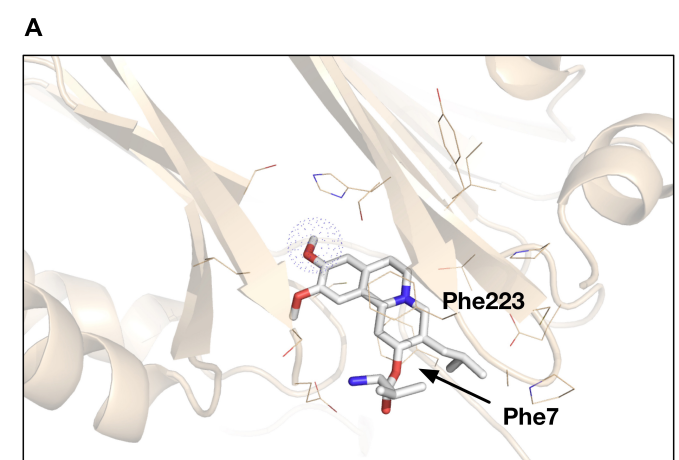

C

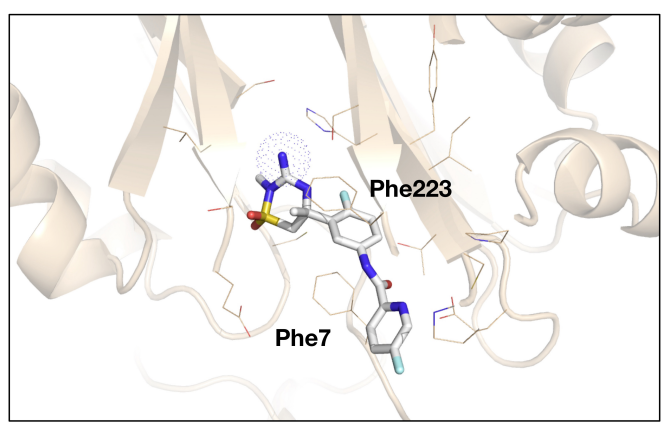

B

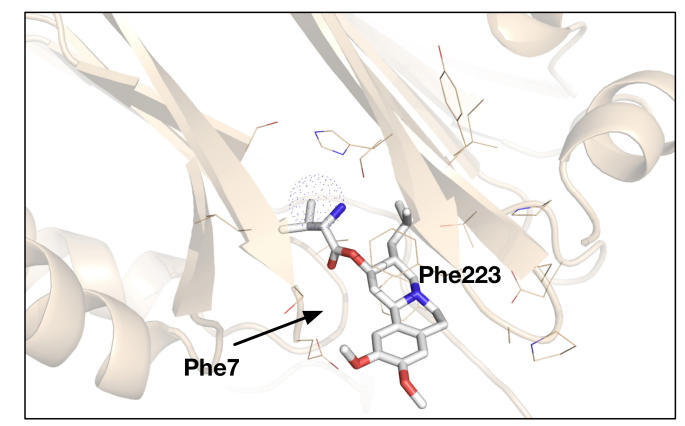

D

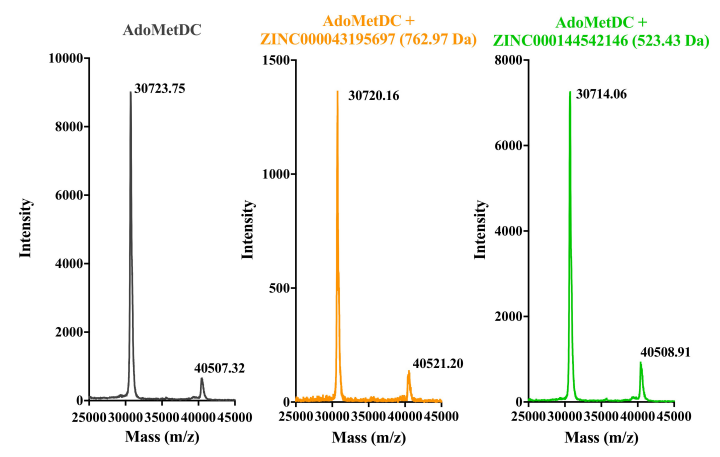

FIGURE 4 | Analyses of the binding conformations and the binding mechanisms of the identified AdoMetDC inhibitors. (A) The overall best (1st) docking conformation of ZINC000043195697 with AdoMetDC. (B) The best (4th) docking conformation of ZINC000043195697 with an amino group close to the covalent center (shown in dotted sphere). (C) The overall best (1st) docking conformation of ZINC000144542146 with AdoMetDC. This is also the best docking conformation with an amino group close to the covalent center. (D) The mass spectrometry (MS) data used to evaluate the covalent binding of the inhibitor and AdoMetDC. The molecular weights of the compounds are shown in parentheses. 


\section{DISCUSSION}

Drug repurposing holds high promise in lowering the economic burden and increasing the success rate in drug development. Both experimental screening approaches and in silico screening approaches are useful in drug repurposing (Cha et al., 2018), but in silico approaches are more economic and could be highly efficient in enriching candidate drugs for experimental validation (Battah et al., 2019). In this work, we extended our SCAR method to the repurposing of clinical drugs as AdoMetDC inhibitors. As demonstrated above, we were able to identify two drugs that can inhibit AdoMetDC's enzymatic activity, which indicates that our screening protocol was successful. However, the false positive signals of ZINC6482036 and ZINC43205655 suggested that the AdoMetDC-PEPC-MDH assay should be performed carefully and coupled with more precise experimental assays such as the HPLC assay.

Two aspects of our in silico screening process proved the high potential of the SCAR strategy in drug repurposing. Firstly, we were able to successfully screen out the known AdoMetDC inhibitor, SAM486A (also known as ZINC000100023874, Sardomozide, or CGP48664) (Pless et al., 2004), from the database. In fact, we used a blind test during the screening process: both the researchers performing the docking process and the researchers manually shortlisting the candidate drugs were not aware of the existence of SAM486A in the database. However, following the rules in this work, this compound was robustly identified by different researchers. Secondly, we successfully validated the inhibitory effects of two compounds in inhibiting AdoMetDC's activity from six compounds. This successful ratio is quite encouraging for the future application of the SCAR strategy on other targets.

As frequently noticed in previous studies, one of the main drawbacks of drug repurposing is that the drug usually has only moderate or very low activity against the new targets (Zheng et al., 2018). This is also true in this work, since the identified compounds only showed moderate to low activities in inhibiting AdoMetDC's activity. One possible reason is the functional groups in ZINC000043195697 and ZINC000144542146 are not active enough to form covalent bonds with Pyr68 of AdoMetDC, so the steric clashes decrease their binding affinities. Nonetheless, there are two possible solutions for this issue. One solution is to use drug combination to increase the potency of this drug (Zheng et al., 2018). For example, combining the identified inhibitors with the inhibitors of polyamine transportation (such as AMXT1501) to lower the effective concentration of the AdoMetDC inhibitors (Gamble et al., 2019). Another solution is to optimize the structure of the identified compounds to improve their activity. However, this solution would indicate more intensive investigations.

Among the two identified AdoMetDC inhibitors, ZINC000043195697 is valbenazine, which is a vesicular monoamine transporter 2 (VMAT2) inhibitor. Valbenazine is the first FDA approved drug for adults with tardive dyskinesia and sold under the trade name Ingrezza. The other one is verubecestat (ZINC000144542146), an inhibitor of beta-secretase 1 treating Alzheimer's disease. Considering that polyamines play important roles in neurodegeneration diseases (Cervelli et al., 2014), their potential role in inhibiting AdoMetDC might be worth further investigations to understand their physiological roles as well as side-effects. For instance, both valbenazine (ThaiCuarto et al., 2018) and verubecestat (Egan et al., 2018) were reported to cause imbalance and falls in the patients, so if this symptom has any relationship with the change of polyamine levels is an interesting question, considering that polyamine levels are decreasing with aging (Minois et al., 2011) but imbalance is usually increasing with aging.

\section{CONCLUSION}

In conclusion, our work demonstrated that, in addition to being useful in de novo drug screening (Ai et al., 2016), the SCAR strategy is also applicable in drug repurposing. Although this work did not find covalent candidates due to the limitations of the database, we hope the SCAR method will find more applications in repurposing both non-covalent and covalent drugs in the future.

\section{DATA AVAILABILITY STATEMENT}

The datasets generated for this study are available on request to the corresponding author.

\section{AUTHOR CONTRIBUTIONS}

SL conceived the idea. SL and QZ did the computational work. SL and YaZ designed the experiments. YaZ did the experimental work. SL, QZ, and YaZ analyzed and interpreted the data. SL, YaZ, and YiZ wrote the manuscript. All authors reviewed and approved the submitted manuscript.

\section{FUNDING}

SL was supported by the grants from the National Natural Science Foundation of China (31670768 and 31971150), the Hubei Provincial Science and Technology Department (2019CFA069), and the Wuhan Science and Technology Bureau of China (2018060401011319).

\section{ACKNOWLEDGMENTS}

We would like to thank the support from the other members in our group and the support from Hubei University of Technology. 


\section{REFERENCES}

Ai, Y., Yu, L., Tan, X., Chai, X., and Liu, S. (2016). Discovery of covalent ligands via noncovalent docking by dissecting covalent docking based on a "Steric-Clashes Alleviating Receptor (SCAR)”. Strategy. J. Chem. Inf. Model. 56, 1563-1575. doi: 10.1021/acs.jcim.6b00334

Baker, N. C., Ekins, S., Williams, A. J., and Tropsha, A. (2018). A bibliometric review of drug repurposing. Drug Discov.Today 23, 661-672. doi: 10.1016/j. drudis.2018.01.018

Battah, B., Chemi, G., Butini, S., Campiani, G., Brogi, S., Delogu, G., et al. (2019). A repurposing approach for uncovering the anti-tubercular activity of FDAApproved drugs with potential multi-targeting profiles. Molecules 24:4373. doi: $10.3390 /$ molecules 24234373

Casero, R. A. (2011). in Polyamine Drug Discovery, 1st Edn, eds P. M. Woster, and R. A. Casero, (London: Royal Society of Chemistry).

Cervelli, M., Angelucci, E., Germani, F., Amendola, R., and Mariottini, P. (2014). Inflammation, carcinogenesis and neurodegeneration studies in transgenic animal models for polyamine research. Amino Acids 46, 521-530. doi: 10.1007/ s00726-013-1572-1573

Cha, Y., Erez, T., Reynolds, I. J., Kumar, D., Ross, J., Koytiger, G., et al. (2018). Drug repurposing from the perspective of pharmaceutical companies. $\mathrm{Br}$. J. Pharmacol. 175, 168-180. doi: 10.1111/bph.13798

Chang, A. Y., Skirbekk, V. F., Tyrovolas, S., Kassebaum, N. J., and Dieleman, J. L. (2019). Measuring population ageing: an analysis of the global burden of disease study 2017. Lancet Public Health 4, e159-e167. doi: 10.1016/S2468-2667(19) 30019-30012

Egan, M. F., Kost, J., Tariot, P. N., Aisen, P. S., Cummings, J. L., Vellas, B., et al. (2018). Randomized trial of verubecestat for mild-to-moderate Alzheimer's disease. N. Engl. J. Med. 378, 1691-1703. doi: 10.1056/NEJMoa170 6441

Gamble, L. D., Purgato, S., Murray, J., Xiao, L., Yu, D. M. T., Hanssen, K. M., et al. (2019). Inhibition of polyamine synthesis and uptake reduces tumor progression and prolongs survival in mouse models of neuroblastoma. Sci. Transl. Med. 11:eaau1099. doi: 10.1126/scitranslmed.aau 1099

Herr, H. W., Warrel, R. P., and Burchenal, J. H. (1986). Phase I trial of alphadifluoromethyl ornithine (DFMO) and methylglyoxal bis (guanylhydrazone) (MGBG) in patients with advanced prostatic cancer. Urology 28, 508-511. doi: 10.1016/0090-4295(86)90154-90158

Hodos, R. A., Kidd, B. A., Shameer, K., Readhead, B. P., and Dudley, J. T. (2016). In silico methods for drug repurposing and pharmacology. Wiley Interdiscip. Rev. Syst. Biol. Med. 8, 186-210. doi: 10.1002/wsbm.1337

Kaitin, K. I. (2010). Deconstructing the drug development process: the new face of innovation. Clin. Pharmacol. Ther. 87, 356-361. doi: 10.1038/clpt. 2009.293

Leaver-Fay, A., Tyka, M., Lewis, S. M., Lange, O. F., Thompson, J., Jacak, R., et al. (2011). ROSETTA3: an object-oriented software suite for the simulation and design of macromolecules. Methods Enzymol. 487, 545-574. doi: 10.1016/B9780-12-381270-4.00019-16
Li, J., Zhang, L., Xiong, J., Cheng, X., Huang, Y., Su, Z., et al. (2019). Polyamines disrupt the KaiABC oscillator by inducing protein denaturation. Molecules 24:3351. doi: 10.3390/molecules24183351

Liao, C., Wang, Y., Tan, X., Sun, L., and Liu, S. (2015). Discovery of novel inhibitors of human S-adenosylmethionine decarboxylase based on in silico high-throughput screening and a non-radioactive enzymatic assay. Sci. Rep. 5:10754. doi: 10.1038/srep10754

Liu, Z., Fang, H., Reagan, K., Xu, X., Mendrick, D. L., Slikker, W., et al. (2013). In silico drug repositioning: what we need to know. Drug Discovery Today 18, 110-115. doi: 10.1016/j.drudis.2012.08.005

Miller-Fleming, L., Olin-Sandoval, V., Campbell, K., and Ralser, M. (2015). Remaining mysteries of molecular biology: the role of polyamines in the cell. J. Mol. Biol. 427, 3389-3406. doi: 10.1016/j.jmb.2015.06.020

Minois, N., Carmona-Gutiérrez, D., and Madeo, F. (2011). Polyamines in aging and disease. Aging (Albany N. Y.) 3, 716-732. doi: 10.18632/aging.100361

Paridaens, R., Uges, D. R., Barbet, N., Choi, L., Seeghers, M., van der Graaf, W. T., et al. (2000). A phase I study of a new polyamine biosynthesis inhibitor, SAM486A, in cancer patients with solid tumours. Br. J. Cancer 83, 594-601. doi: 10.1054/bjoc.2000.1305

Pegg, A. E. (2009). Mammalian polyamine metabolism and function. IUBMB Life 61, 880-894. doi: 10.1002/iub.230

Pless, M., Belhadj, K., Menssen, H. D., Kern, W., Coiffier, B., Wolf, J., et al. (2004). Clinical efficacy, tolerability, and safety of SAM486A, a novel polyamine biosynthesis inhibitor, in patients with relapsed or refractory non-Hodgkin's lymphoma: results from a phase II multicenter study. Clin. Cancer Res. 10, 1299-1305.

Scannell, J. W., Blanckley, A., Boldon, H., and Warrington, B. (2012). Diagnosing the decline in pharmaceutical R\&D efficiency. Nat. Rev. Drug Discov. 11, 191-200. doi: 10.1038/nrd3681

Sterling, T., and Irwin, J. J. (2015). ZINC 15 - ligand discovery for everyone. J. Chem. Inf. Model. 55, 2324-2337. doi: 10.1021/acs.jcim.5b00559

Thai-Cuarto, D., O’Brien, C. F., Jimenez, R., Liang, G. S., and Burke, J. (2018). Cardiovascular profile of valbenazine: analysis of pooled data from three randomized, double-blind, placebo-controlled trials. Drug Saf. 41, 429-440. doi: 10.1007/s40264-017-0623-1

Zheng, W., Sun, W., and Simeonov, A. (2018). Drug repurposing screens and synergistic drug-combinations for infectious diseases. Br. J. Pharmacol. 175, 181-191. doi: 10.1111/bph.13895

Conflict of Interest: The authors declare that the research was conducted in the absence of any commercial or financial relationships that could be construed as a potential conflict of interest.

Copyright (ㄷ) 2020 Zhang, Zheng, Zhou and Liu. This is an open-access article distributed under the terms of the Creative Commons Attribution License (CC BY). The use, distribution or reproduction in other forums is permitted, provided the original author(s) and the copyright owner(s) are credited and that the original publication in this journal is cited, in accordance with accepted academic practice. No use, distribution or reproduction is permitted which does not comply with these terms. 